\title{
COMPARATIVE ANALYSIS OF FLUID COOLING SYSTEMS IN MOTORIZED SPINDLES
}

\author{
L. Koch ${ }^{1 *}$, K. Gross ${ }^{1}$, G. Krueger ${ }^{1}$ \\ ${ }^{1}$ University of Applied Sciences Wuerzburg-Schweinfurt, Laboratory of Machine Tools, Schweinfurt, Germany \\ *Corresponding author; e-mail: Lukas.koch@fhws.de
}

\begin{abstract}
This paper analyzes geometrical approaches to optimize the fluid cooling circulation of motorized spindles. The spindle fluid cooling's effectiveness, efficiency and influence on the machine's precision are analyzed through observations of the stator temperature, pressure drop and thermal asymmetry, respectively. The observation is based on a validated coupled thermal/fluid mechanical simulation model. The widely used helix and meander shape stator cooling sleeves are primarily investigated. Additionally, a so-called S-meander shape was developed, which combines the advantages of the formerly mentioned sleeves. In order to understand the nonlinear thermal interactions properly, width and height of the cooling channels were varied separately and simultaneously. While keeping the flow rate identical, the average stator temperature could be decreased by $2.3 \mathrm{~K}$ solely with geometrical optimizations. Interestingly, the motor temperature is not continuously decreased by raising the fluid velocity through a reduction of the cooling channels size. For the helix and the S-meander, the temperature actually increases after passing a certain geometrical sweet spot. Additionally, this optimum is different for the helix, meander and Smeander cooling sleeve. The results imply that the geometrical optimization of fluid cooling channels in motorized spindles has a significant potential. Furthermore, the developed cooling sleeves are transferable to any electric motor with fluid cooling.
\end{abstract}

\section{Keywords:}

Motor spindle; cooling; electric motor; machine tools

\section{INTRODUCTION}

The increase in productivity has heightened the need for effective cooling systems in machine tools. The cooling of the motorized spindle is of particular interest, as overheating issues occur most frequently in the spindle. For this reason, motorized spindles usually require separate fluid cooling systems, absorbing the heat dissipated from the bearings and the electric motor inside the spindle.

Subsequently, creating better cooling systems for motorized spindles became an important research field. Usually coupled thermal/fluid-mechanical simulation models are required to generate accurate temperature fields. The fluid cooling itself is usually realized through a computational fluid dynamics (CFD) simulation. Zhen-nan analyzed the cooling performance of a helix, axial meander, circumferential and a half helical shaped cooling sleeve for electric motors [Zhen-nan 2011]. The sleeves' evaluation was based on pressure drop, temperature difference between fluid/wall and the heat transfer coefficient. Pechanek simulated a fluid cooling of an electric motor [Pechanek 2012]. A tangential meander shaped and an axial meander shaped cooling sleeve were simulated. As evaluation criterion the heat transfer coefficient, pressure drop, fluid inlet/outlet temperature, the sleeves temperature and hot spots were considered. Ganesh researched the cooling of a helix, double helix and axial meander shaped cooling sleeve in motorized spindles [Ganesh 2012]. The performance analysis of the cooling systems was based on heat transfer coefficient and pressure drop. Weber observed the cooling of a motorized spindle empirically and based on simulation models [Weber 2016]. Both a classic helix and a less common double helix cooling sleeve were observed. The simulation results were discussed based on the pressure drop and the temperature increase of the fluid.

The literature shows that evaluating fluid circulation systems without a certain geometry variation only gives a limited understanding of its cooling potential. Yang therefore varied the cooling channel dimensions (height, width) of a helix shaped stator cooling sleeve and evaluated it based on wall temperature and pressure drop [Yang 2016]. Cuiping also analyzed the variation of the cooling channel height and width [Cuiping 2017]. Furthermore, the adjacent waterway of the observed meander shaped sleeve was varied in three steps. The designs were compared based on motor temperature and pressure drop. Satrustegui observed a helix, U-shaped, meander and axial meander shaped cooling sleeve [Satrustegui 2017]. The parameter variation included channel height, width, different distances, thermal resistances, shaft diameter and housing material. The results were evaluated based on stator temperature. 
The inconsistency regarding the evaluation criteria is obvious. Furthermore, the authors usually do not add any explanation why certain criteria were chosen. The comparability of studies with geometrical variation is further restricted by differences in result presentations. As no consensus regarding essential observation criteria and their presentation exists, the studies' comparability is severely restricted.

This paper presents an evaluation concept with the aim to make comparative analysis easier. According to preliminary investigations of the author team, a motor spindle cooling sleeve's performance can be evaluated based on the following three pillars: effectiveness (motor temperature), efficiency (pressure drop) and influence to the machine's precision (thermal asymmetry). Motor temperature and pressure drop were investigated previously, yet the cooling system's influence on the machine's precision through thermal asymmetry was ignored. Nevertheless, thermal asymmetry is commonly regarded as an essential machine design criterion [Brecher 2018, Horejs 2012, Liu 2016, Abele 2010]. A simultaneously published paper [Koch 2021] presents a novel formulation to quantify thermal asymmetry. Furthermore, it shows how the thermal asymmetry caused by the cooling system offsets the tool center point radially.

The developed evaluation concept is applied to the most common stator cooling sleeves: the helix and the meander shaped sleeve. Based on this observation, a third stator cooling, the S-meander shaped sleeve, is developed. In order to evaluate the cooling sleeves comprehensively, a parameter variation of the cooling channel's width and height is conducted and the results are compared based on pressure drop, thermal asymmetry and stator temperature.

\section{BASIS OF THE EVALUATION}

The investigation is based on a previously published simulation model which is explained in chapter 2.1. The results in the subsequent chapters 3 and 4 are based on this model and evaluated based on the criteria introduced in chapter 2.2 .

\subsection{Simulation model}

The previously published coupled thermal/fluid-mechanical simulation model with its boundary conditions is shown in Fig. 1 [Koch 2017]. The boundary conditions are divided into heat sources, heat sinks and heat transfer systems. The main heat source is the electric motor which is represented by separate boundary conditions for rotor and stator. Additionally, the four rolling bearings are represented as heat source. As explained in [Koch 2017], the bearings' heat generation was determined empirically, as the approaches currently available to calculate bearing heat are not accurate under most operating conditions. The observed heat transfer systems include the bearing geometries and the gap between shaft and housing. The four bearings are not represented by their models but respective heat transitions coefficients. In order to determine the heat transition between the shaft and the housing realistically, the area was geometrically divided into nine parts. For each of these housing and shaft geometry diameters combinations a separate heat transition coefficient was calculated. Lastly, both the ambient air and the fluid cooling were represented as heat sink. To represent the ambient air realistically, the spindle's surface was divided into separate parts with forced and free convection. The fluid cooling inside the spindle's housing was represented as coupled CFD simulation. This representation is a necessity for this analysis, as the generated temperature

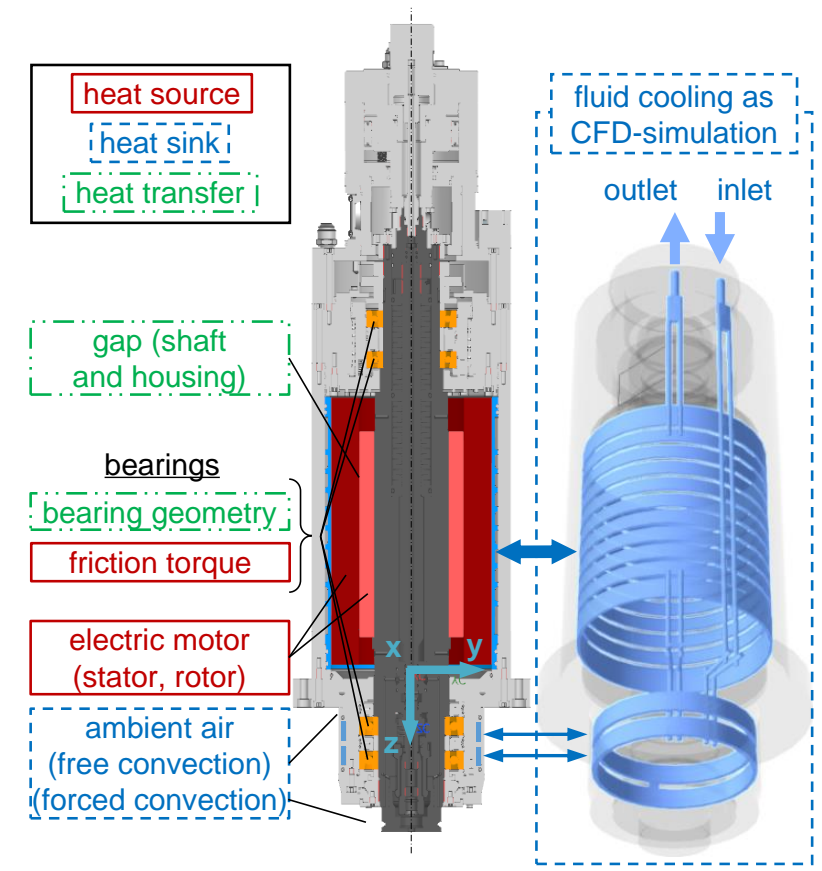

Fig. 1: Boundary condition of the simulation model based on [Koch 2017].

field becomes realistic through coupling the thermal analysis to a CFD simulation. The conducted thermal asymmetry analysis, which is explained in the subsequent chapter 2.2, does require these accurate temperature fields of coupled simulation models in particular.

The fluid flow at the inlet of the fluid cooling system is set to $3 \mathrm{l} / \mathrm{min}$. The $\mathrm{k}-\varepsilon$ turbulence model was used with water as fluid material. The mesh of the fluid circulation system was refined until its $Y_{+}$values reached a reasonable range [Anderl 2014]. The simulation model was validated based on readings with the real spindle equipped with PT-100 temperature sensors on its surface. The temperature difference of the spindle in idle mode after 5 hours and the model in thermal equilibrium was $0.8^{\circ} \mathrm{C}$ on average.

The cooling system's performance can only be evaluated properly under load. Therefore, the simulation model was adjusted in order to represent the spindle under load. The input power thus changed from $815 \mathrm{~W}$ to $25,000 \mathrm{~W}$ for the analysis in chapter 3 and 4. 25,000 W is the electric motor's maximum input power for a continuous operation. Accordingly, the power loss of the electric motor changed from $57 \mathrm{~W}$ to $1,750 \mathrm{~W}$. The power loss of the bearings is primarily caused by its rotation speed. Therefore, it was kept identical at $758 \mathrm{~W}$. The calculation approaches of the boundary conditions are described in detail in a previous paper of the author team [Koch 2017].

\subsection{Evaluation criteria}

The evaluation of fluid cooling systems in motorized spindles requires appropriate evaluation criteria. In the aforementioned literature (chapter 1), different criteria were observed as a basis of evaluation. There is no consensus regarding the most relevant evaluation criteria. One of the main focuses of the preliminary investigation of the author team was the identification of the most relevant criteria for a comparative analysis of fluid cooling systems in motorized spindles. Therefore, 30 simulation models with different fluid cooling systems were developed based on the validated simulation model in the previous chapter. In order to get a better overview, each of the following criteria were analyzed with regard to the results of the 30 simulation models: 
1. required input power for the pump;

2. coefficient of performance (COP);

3. pressure drop between the inlet to the outlet of the fluid;

4. axial tool center point (TCP) displacement;

5. radial tool center point (TCP) displacement;

6. thermal asymmetry of the stator;

7. thermal asymmetry of the housing;

8. average fluid velocity;

9. heat transfer coefficient between the fluid and the surrounding housing;

10. fluid areas with extremely low velocity (death areas);

11. housing temperature;

12. shaft temperature;

13. front bearing temperature;

14. rotor temperature;

15. stator temperature

For the evaluation, the 15 criteria are split into three groups. The first group (criteria 1.-3.) considers the efficiency with regard to the energy consumption of the pump. The second group (criteria 4.-7.) assesses the cooling system's influence to the precision of the machine. The third one (criteria 8.-15.) considers the temperature condition and thereby the effectiveness of the cooling systems.

In the preliminary investigation, all three efficiency criteria (1.-3.) of the system were observed. Both the pump's input power (1.) and the COP (2.) include the pressure drop (3.). Furthermore, the input power requires an electric (motor) and hydraulic (pump) efficiency value, which can vary greatly, yet are of no interest for the evaluation of fluid cooling systems itself. The flow rate is also required for the calculation of the input power. However, as the flow rate is kept identical for comparative reasons, it is irrelevant as well. The COP calculation includes the temperature increase of the fluid. Surprisingly, the temperature increase is almost identical for most of the observed fluid geometries. The fluid cooling always remains the main heat sink of the motorized spindle, the heat is just absorbed at different temperature values. Thus the pressure drop (3.) is decisive for both the COP and the input power and the most relevant criterion for evaluating the system's efficiency.

The criteria (4.-7.) estimating the cooling system's influence on the precision of the spindle were also compared. The spindle's precision is reduced through an axial (4.) and radial (5.) TCP displacement. The axial displacement decreases when the effectiveness of the stator cooling system increases. However, the preliminary investigation with thermomechanical spindle models showed that the reduction of axial TCP displacement remains small: Increasing the effectiveness of the cooling system in the spindle's housing did not decrease the axial TCP displacement by more than $5.3 \%$. However, the radial TCP displacement can be reduced significantly through cooling system adjustments. The core problem lays within the thermal asymmetry of the spindle's housing (7.). How the housing's thermal asymmetry offsets the TCP is explained in detail in a paper published simultaneously [Koch 2021]. The study showed that cooling channel adjustments can decrease the radial TCP displacement by $97.4 \%$. For this reason, both the stator's and the housing's average thermal asymmetry $\boldsymbol{\tau}_{(z)}$ are calculated with equation (1):

$\boldsymbol{\tau}_{(z)}=\sqrt{x_{(z)}^{2}+y_{(z)}^{2}}$
Here, $x_{(z)}$ and $y_{(z)}$ are the arithmetic averages of the thermal asymmetry terms $x_{d(z)}$ and $y_{d(z)}$ for discs $d_{(z)}$ along the Z-axis. The Z-axis is the longitudinal axis as visualized in Fig. 1. Therefore, the term $\boldsymbol{\tau}_{(z)}$ is a measure for the thermal asymmetry in radial direction along this axis. Its unit is $\mathrm{m}$. A higher value indicates higher thermal asymmetry, which leads to more angular/radial displacement. The complete derivation of $\boldsymbol{\tau}_{(z)}$ is presented in [Koch 2021].

The housing's asymmetry is generally a more valid measure than the stator asymmetry (6.), as the housing's radial displacement directly causes the radial TCP offset. Therefore, observing the housing's thermal asymmetry is a legitimate way to estimate the radial TCP displacement. Additionally, calculating the thermal asymmetries does not require further complex thermo-mechanical simulation models.

The evaluation of the cooling system's effectiveness (criteria 8.-15.) is extremely inconsistent in the literature. In the preliminary observation the fluid channel characteristics were evaluated. However, neither an increased fluid velocity (8.) nor an enhanced heat transfer coefficient (9.) showed a consistently improved cooling effect. Areas with extremely low fluid velocity (10.) increased thermal asymmetry and decreased the heat transfer locally. Their effect on the cooling effectiveness however remained small and inconsistent for the observed fluid systems. Therefore, judging the cooling effectiveness by the resulting temperatures of the individual components seems to be the most reasonable way. Cooling systems with a good cooling performance usually also decrease the housing (11.), shaft (12.), front bearing (13.), rotor (14.) and stator (15.) temperature. The electric motor is generally the first part with overheating issues in motorized spindles. Primarily the stator overheats due to a bad heat dissipation in the area of the winding overhangs. The stator of the electric motor is modeled as a hollow cylinder in the current iteration of the model (chapter 2.1). The problematic winding overhangs are thus not modelled accurately. In future research, the model should be enhanced by a geometrically extremely accurate electric motor. However, the preliminary investigation showed that the average stator temperature correlates with its maximum temperature. For this reason, the observation of the average stator temperature is legitimate for the evaluation of cooling systems. Observing average temperatures of finite element simulation models often leads to distorted results, as the mesh density varies locally. Locally dense meshes have a higher impact on averaged results. For this reason, an algorithm was written which divides the stator's temperature field into ten discs along its longitudinal axis. Each of these discs is then subdivided into four volume elements. The stator node temperatures are allocated to their respective volume element through the individual node locations. Based on that, the arithmetic average temperature of each volume element, disc and the entire stator is calculated. This approach greatly decreases the influence of local mesh variations.

In summary, this concept reduces the number of evaluation criteria to three essential ones. These include measures to evaluate the efficiency, the influence on the machine's precision and the effectiveness of the cooling system:

1. pressure drop between the inlet and the outlet of the fluid (efficiency);

2. thermal asymmetry of the housing (precision);

3. stator temperature (effectiveness)

This evaluation concept was developed for cooling systems in motorized spindles. Nevertheless, it should be transferable to other components of the machine tool with fluid 
cooling (rotary table, machine bed etc.). In general, the three pillar concept remains valid for cooled components with high precision requirements. However, the stator temperature might be replaced by a different temperature for applications without an electric motor.

These three criteria are the basis of the fundamental observation of spindle cooling systems in chapter 3 . Moreover, each of these criteria is observed individually in chapter 4.1 (efficiency), 4.2 (precision) and 4.3 (effectiveness) in order to carry out a substantial comparative analysis of cooling systems with geometrical variation.

\section{BASIC SHAPE ANALYSIS OF THE STATOR COOLING SLEEVE}

This paper presents a comparative analysis of fluid cooling systems in motorized spindles. However, there are more than 30 different basic shapes of stator cooling sleeves (helix, u-shaped, circumferential, meander, axial meander etc.). In the preliminary investigation most of them were evaluated with the 15 criteria listed in the previous chapter. Many of the newer, more innovative designs either had issues with high thermal asymmetry, pressure drop or temperature. While keeping the fluid flow rate identical, the average stator temperature varied between $62.9^{\circ} \mathrm{C}$ (circular divided shape, see the second sleeve in Fig. 2 in [Weber 2016]) and $45.6{ }^{\circ} \mathrm{C}$ (helix, see Fig. 2 a)). The conventional helix and the meander shaped stator cooling sleeve usually showed the best overall performance and will be discussed in detail in the next chapter. All the presented numbers show the simulation results in thermal equilibrium.

\subsection{Helix and meander shaped stator cooling sleeve}

A conventional helix shaped stator cooling sleeve is visualized in Fig. 2 a). The fluid, this coming from the front bearings, enters the sleeve through two drillings. It enters the inlet area. This area, existing solely due to manufacturing reasons, is the main source of the problem of this sleeve. Most of the fluid flows right into the helix. Therefore,

the fluid circulates the inlet area slowly, resulting in bad cooling performance and thermal asymmetry. The outlet area essentially has the same problem. Weber discussed the problem of these areas in detail [Weber 2013]. In contrast, the helix itself generates one of the best cooling effects, as it features high velocity and minimal temperature gradients between successive coils.

While the conventional helix usually sees most use in practice, spindles with meander shaped sleeves (Fig. 2 b)) have become more numerous in recent years. The construction is quite simple, as it generally features a number of identical rings. Few millimeters of every second ring are milled off to create a transition flow. Likewise, the remaining rings are milled off on the opposite side of the sleeve. The fluid coming from the two drillings circulates each ring and passes over to the next ring. Each ring is circulated on each side simultaneously. Therefore, the fluid flows with half the velocity through the meander shaped sleeve compared to the other sleeves. That usually results in a decreased cooling performance.

Tab. 1 compares the aforementioned sleeves. All sleeves are shown in the same basic configuration, featuring a channel height of $3 \mathrm{~mm}$ and 10 rings or windings. The

Tab. 1: Comparative analysis of a helix, meander and $S$-meander shaped stator cooling sleeve.

helix meander S-meander

\begin{tabular}{lrrr}
\hline $\begin{array}{l}\text { channel height } \\
\text { number of rings }\end{array}$ & $\begin{array}{r}3 \mathrm{~mm} \\
10\end{array}$ & $\begin{array}{r}3 \mathrm{~mm} \\
10\end{array}$ & $\begin{array}{r}3 \mathrm{~mm} \\
10\end{array}$ \\
\hline $\begin{array}{l}\text { pressure drop } \\
\text { (efficiency) }\left[10^{5} \mathrm{~Pa}\right]\end{array}$ & 0.53 & 0.47 & 0.35 \\
$\begin{array}{l}\text { thermal asymmetry } \\
\text { (precision) [mm] }\end{array}$ & 2.38 & 2.44 & 2.00 \\
$\begin{array}{l}\text { stator temperature } \\
\left.\text { (effectiveness) [ }{ }^{\circ} \mathrm{C}\right]\end{array}$ & 45.63 & 46.38 & 45.38 \\
\hline
\end{tabular}
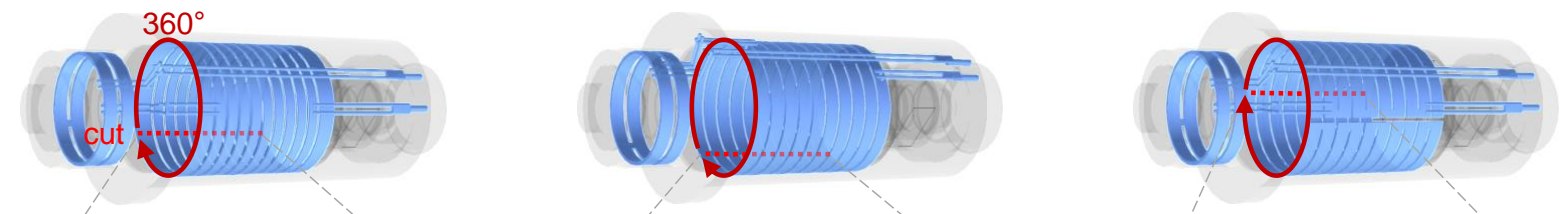

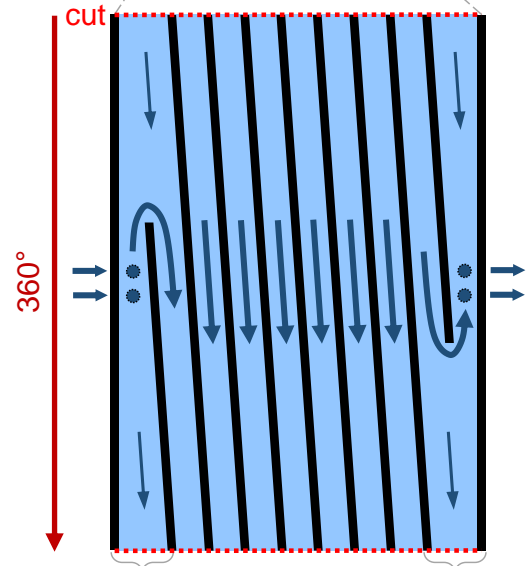

inlet area outlet area

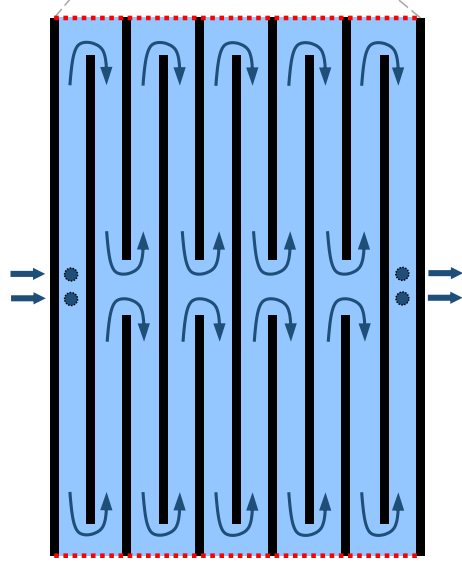

(b) meander shaped cooling sleeve

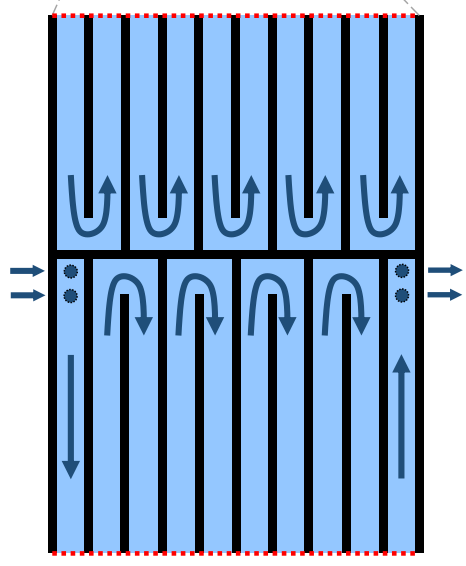

(c) S-meander shaped cooling sleeve

(a) helix shaped stator cooling sleeve

Fig. 2: The three observed stator cooling sleeves unrolled. The fluid coming from the front bearing enters the stator cooling sleeve through two drillings, passes the sleeve's respective geometry and exits through a second set of drillings. 
channel height and the number of rings are visualized in Fig. 3 for clarification. The channel's width is not observed separately, as it decreases with an increasing number of rings.

The meander shaped cooling sleeve has a lower pressure drop and thus might reflect a more efficient cooling sleeve (Tab. 1). In these configurations, the conventional helix features a lower thermal asymmetry and should thus also feature a lower radial TCP displacement (see [Koch 2021]). The average stator temperatures are calculated according to the approach in chapter 2.2. The helix cools the stator significantly better than the meander shaped sleeve, most likely due to the problem of lowered fluid velocity of the latter. Nevertheless, the table shows the results only for configurations with $3 \mathrm{~mm}$ channel height and 10 rings. The analysis in chapter 4 reveals that the individual results can be altered significantly through geometrical variation.

\subsection{S-meander shaped stator cooling sleeve}

An analysis of the advantages of the helix and meander shaped cooling sleeves resulted in the development of the $\mathrm{S}$-meander (Fig. $2 \mathrm{c}$ )). The main advantage of the helix is its high fluid velocity, while the inlet and outlet areas lead to high pressure drop and create thermal asymmetry. The meander does not have the problematic transitions (inlet/ outlet) and thus features a lower pressure drop but has a low fluid velocity. The basic idea of the S-meander is to send all the fluid through one channel, just like the helix, creating high fluid velocity. This is achieved by coiling the channel in S-shapes around the sleeve. The fluid circulates, as visualized in Fig. $2 \mathrm{c}$ ), almost $360^{\circ}$ around the stator. The transition to the next ring is then realized just like in the meander shaped sleeve. The fluid then flows back almost $360^{\circ}$ to enter the next ring. The sleeve is comparable to the meander, while the velocity in the rings is as high as in the helix, because the fluid flows through one single channel. The name S-meander originates from its fluid circulation.

The simulation results of the S-meander are displayed in Tab. 1. The design shows a lower pressure drop, thermal asymmetry and stator velocity compared to the helix and the meander. Surprisingly, Satrústegui [Satrústegui 2017] and Zhen-nan [Zhen-nan 2011] actually discussed comparable designs, yet they did not recognize their potential. Therefore, further research was required in order to get a better picture of its potential. This design will also be featured in the geometrical variation in chapter 4.

\section{GEOMETRICAL VARIATION OF THE STATOR COOLING SLEEVES}

In this chapter the three basic stator cooling sleeves from the previous chapter are analyzed in detail. The cooling channel height was changed in $1 \mathrm{~mm}$ steps from 1 to $3 \mathrm{~mm}$ (see Fig. 3). The number of rings was varied from 10 to 20 and finally 30 rings, while the total length of the sleeve remained always identical. This variation was done separately and simultaneously for the helix (Fig. 2 a)), meander (Fig. 2 b)) and s-meander (Fig. 2 c)) shaped stator cooling sleeve, creating nine coupled thermal/fluidmechanical simulation models for each, and adding up to 27 simulation models in total. Their simulation time varied between 20 and 40 hours.

The simulation results are observed in thermal equilibrium. They are discussed based on the criteria derived in chapter 2.2. In the first step, the pressure drop values are compared (chapter 4.1). In chapter 4.2 the thermal asymmetries are analyzed. Finally, the average stator temperatures are compared in chapter 4.3.

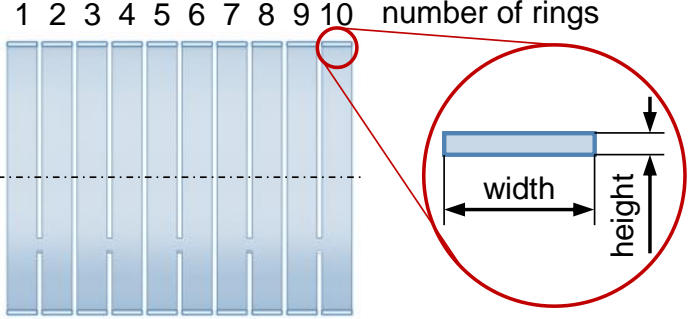

Fig. 3: The channel height and the number of rings are varied. The width decreases with an increasing number of rings. The example shows the meander shaped sleeve, but the approach is identical for all sleeves.

\subsection{Pressure drop analysis (efficiency)}

Tab. 1 compares the pressure drop values of $3 \mathrm{~mm}$ channel height and 10 ring configurations. This configuration is visualized on the lower end of each observation in Fig 4a. A closer look at the pressure drop of the helix shaped sleeve (Fig. 4 a)) reveals that decreasing the height and increasing the number of rings leads to a higher pressure drop. This is especially true for the sleeve with $1 \mathrm{~mm}$ and 30 rings. Its pressure drop rose up to $144.1 \cdot 10^{5} \mathrm{~Pa}$, representing the highest of all observed pressure drops. The helix reaches its lowest value at $3 \mathrm{~mm}$ and 10 rings with $0.53 \cdot 10^{5} \mathrm{~Pa}$.

The graph for the meander shaped sleeve in Fig. 4 b) reveals the biggest advantage of the meander shaped design: the pressure stays comparatively low although the channel size decreases. It peaks in the configuration of $1 \mathrm{~mm}$ channel height and 20 rings, reaching $8.7 \cdot 10^{5} \mathrm{~Pa}$. The configuration with $3 \mathrm{~mm}$ and 10 rings achieves the lowest value $\left(0.47 \cdot 10^{5} \mathrm{~Pa}\right)$.

The pressure drop of the S-meander (Fig. $4 \mathrm{c}$ )) is comparable to the helix. It rises significantly in the tightest configuration of $1 \mathrm{~mm}$ and 30 rings, peaking at $120.1 \cdot 10^{5} \mathrm{~Pa}$. Just like the aforementioned helix, the S-meander has the lowest pressure drop in the configuration of $1 \mathrm{~mm}$ and 10 rings $\left(0.35 \cdot 10^{5} \mathrm{~Pa}\right)$. Surprisingly, this single value is even lower than the lowest value of the meander shaped sleeve.

The observation shows that the meander features the lowest pressure drops in most configurations. The helix and the newly developed S-meander are generally comparable, while the helix gives slightly higher results.

\subsection{Thermal asymmetry observation (precision)}

Thermal asymmetry is caused by the individual flow characteristics and the cooling attributes of the fluid circulation system. The spindle housing's thermal asymmetry causes the radial TCP displacement (see chapter 2.2).

The thermal asymmetry introduced by the helix shaped sleeve (Fig. 5 a)) can be reduced by thinner cooling channels and a higher number of rings. Generally it works best in 30 ring configurations. With 30 rings it has an average asymmetry of $1.23 \mathrm{~mm}, 1.15 \mathrm{~mm}$ and $1.15 \mathrm{~mm}$ for a fluid channel height of $3 \mathrm{~mm}, 2 \mathrm{~mm}$, and $1 \mathrm{~mm}$, respectively. In other words, thermal asymmetry can be halved through stator cooling sleeve optimization (see Fig. 4a and Tab. 1).

The meander shaped sleeve's (Fig. 5 b)) thermal asymmetry is a little higher, but otherwise it performs very similar to the helix shape. It is slightly less sensitive to reduced channel width. The lowest thermal asymmetry results are 


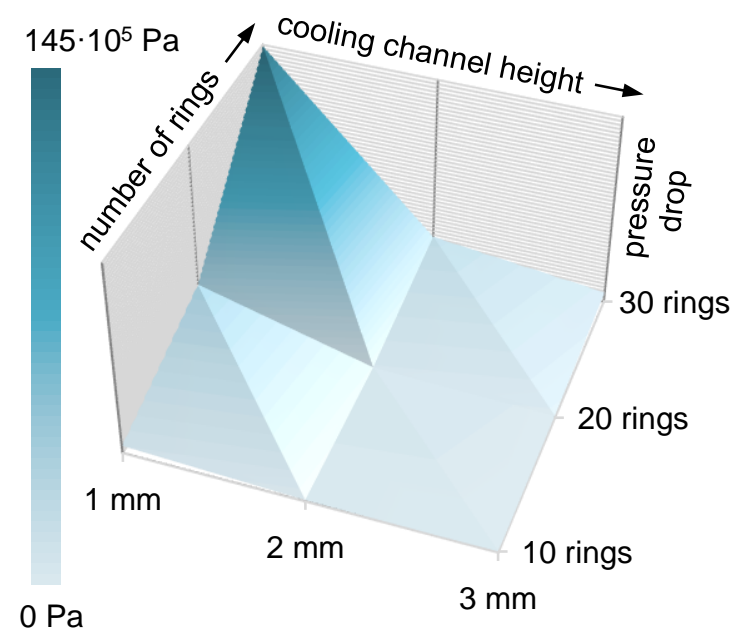

(a) helix stator cooling sleeve

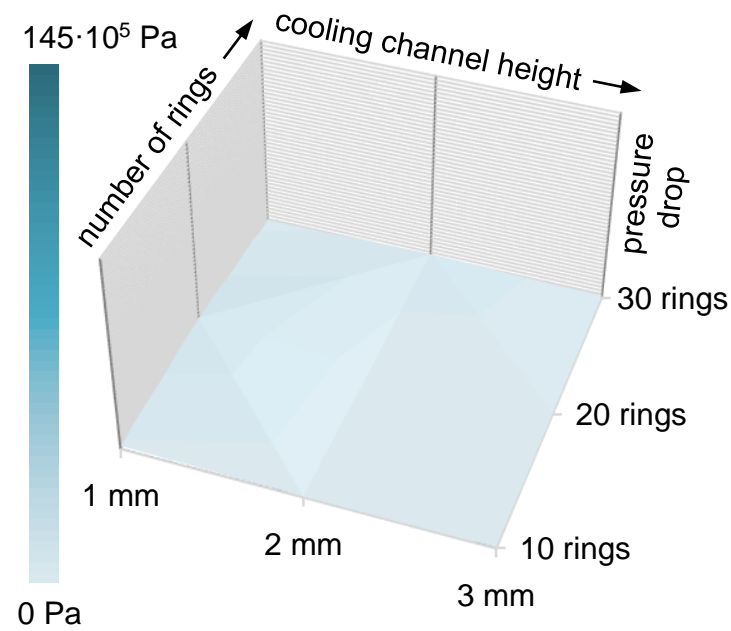

(b) meander stator cooling sleeve

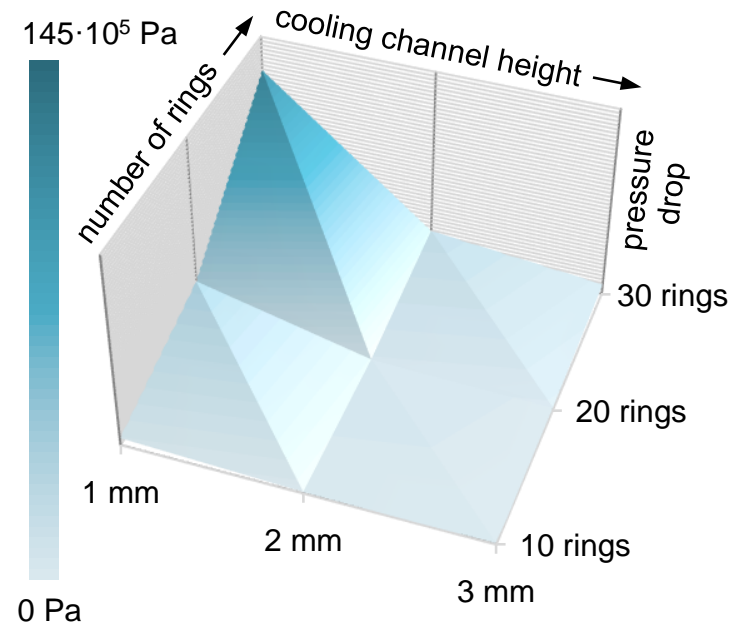

(c) S-meander stator cooling sleeve

Fig. 4: Pressure drop graphs through a parameter variation of a helix shaped (Fig. 4 a)), meander shaped

(Fig. 4 b)) and S-meander shaped (Fig. 4 c)) stator cooling sleeve's number of rings and channel height. obtained with 30 rings. In this configuration, the thermal asymmetry reaches $1.35 \mathrm{~mm}, 1.22 \mathrm{~mm}$ and $1.22 \mathrm{~mm}$ for a channel height of $3 \mathrm{~mm}, 2 \mathrm{~mm}$ and $1 \mathrm{~mm}$, respectively.

The S-meander cooling sleeve's asymmetry is significantly lower in comparison to the meander and helix shaped sleeves. As visualized in Fig. $5 \mathrm{c}$ ), the thermal asymmetry reaches values below $1 \mathrm{~mm}$. The lowest thermal asymmetry of $0.86 \mathrm{~mm}$ is achieved by the configuration with 30 rings and $2 \mathrm{~mm}$. Making the cooling channel even thinner (30 rings, $1 \mathrm{~mm}$ height) increases the asymmetry to $0.93 \mathrm{~mm}$. Most probably, this effect is directly linked to the decreased cooling performance of this configuration $(1 \mathrm{~mm}$ and 30 rings) documented in chapter 4.3 .

The simultaneously published observation [Koch 2021] shows that a better cooling performance generally equals a lower thermal asymmetry in the housing. Most probably this is the reason for the lower thermal asymmetries of configurations with thinner channels. This effect might also explain the increased thermal asymmetry for the Smeander configuration with $1 \mathrm{~mm}$ and 30 rings.

\subsection{Stator temperature analysis (effectiveness)}

Cooling systems should cool as effectively as possible. Thus, the average temperature values of the observed stator in electric motors can be regarded as the most important criterion. They are calculated based on the approach explained in chapter 2.2.

As visualized in Fig. 6 a), the helix shaped cooling sleeve shows the best result for the configuration with 30 rings and $2 \mathrm{~mm}\left(44.28^{\circ} \mathrm{C}\right)$. Surprisingly, the cooling effect deteriorated for the thinnest cooling channel (1 $\mathrm{mm}, 30$ rings). One likely explanation for this phenomenon is the heating of the fluid itself. The fluid channel size reduction decreased the fluid's volume. Therefore, the fluid heats up quicker, decreasing its cooling performance. At this point, the temperature $\left(45.65^{\circ} \mathrm{C}\right)$ is higher than in the initial configuration $\left(45.63^{\circ} \mathrm{C}\right)$. The graph actually shows a valley between the configuration to the very left (1 $\mathrm{mm}$ and 10 rings) and the very right ( $3 \mathrm{~mm}$ and 30 rings). This was confirmed through two additional simulation models with $1.5 \mathrm{~mm} / 30$ rings and $1 \mathrm{~mm} / 25$ rings. The configuration at $3 \mathrm{~mm}$ and 30 rings also showed good results $\left(44.41^{\circ} \mathrm{C}\right)$. The helix reaches its sweet spot in this spindle with a configuration of more than 30 rings and about $2 \mathrm{~mm}$ channel height.

The meander shaped cooling sleeve temperatures displayed in Fig. 6 b) show a completely different performance compared to the other two sleeves. As Tab. 1 suggests, it is much worse in the initial configuration, yet it yields excellent results $\left(44.15^{\circ} \mathrm{C}\right)$ with the thinnest fluid channel (1 mm, 30 rings). Surprisingly, in its best configuration the meander cools better than the helix. The graph suggests that the meander's cooling performance can be improved further. Most probably it reaches its sweet spot with a configuration below $1 \mathrm{~mm}$ channel height and about 30 rings.

The results of the S-meander shaped stator cooling sleeves are displayed in Fig. $6 \mathrm{c}$ ). In the range of examined variants, it generally created the best results. The stator temperature is as low as $44.15^{\circ} \mathrm{C}$ for the configuration at $1 \mathrm{~mm}$ and 10 rings already. The configuration with $1 \mathrm{~mm}$ and 20 rings is just a little worse $\left(44.18^{\circ} \mathrm{C}\right)$. With $44.06^{\circ} \mathrm{C}$, the configuration at $2 \mathrm{~mm}$ and 30 rings yields the best result of all observed models. Furthermore, the model with $3 \mathrm{~mm}$ and 30 rings gives the second-best result at $44.08^{\circ} \mathrm{C}$. The S-meander's sweet spot is thus expected at more than 30 rings and about $2.5 \mathrm{~mm}$ channel height. However, there 


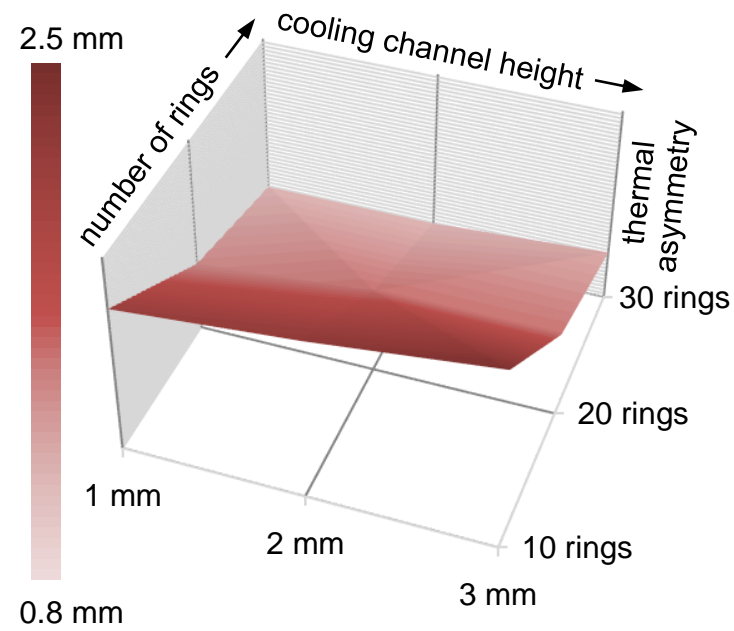

(a) helix stator cooling sleeve

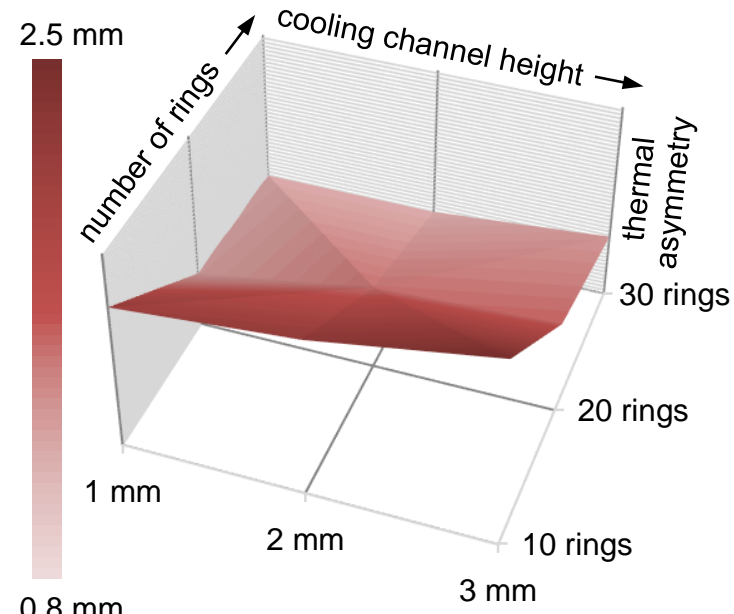

(b) meander stator cooling sleeve

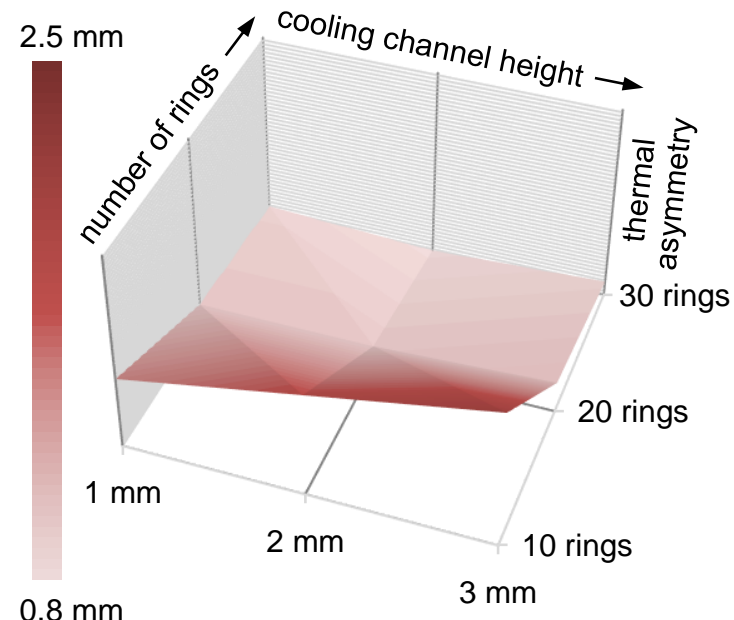

(c) S-meander stator cooling sleeve

Fig. 5: Average thermal asymmetry $\boldsymbol{\tau}_{(z)}$ of the spindle housing through a parameter variation of a helix shaped (Fig. 5 a)), meander shaped (Fig. 5 b)) and S-meander shaped (Fig. 5 c)) stator cooling sleeve's number of rings and channel height.

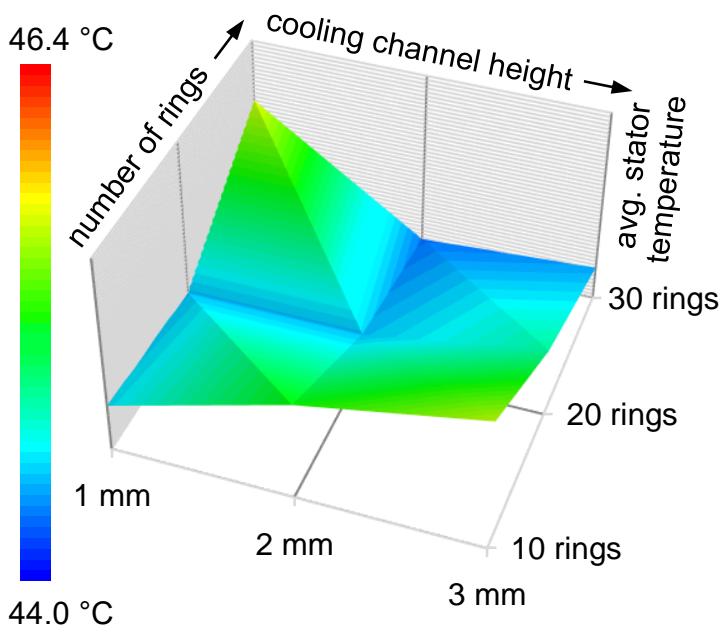

(a) helix stator cooling sleeve

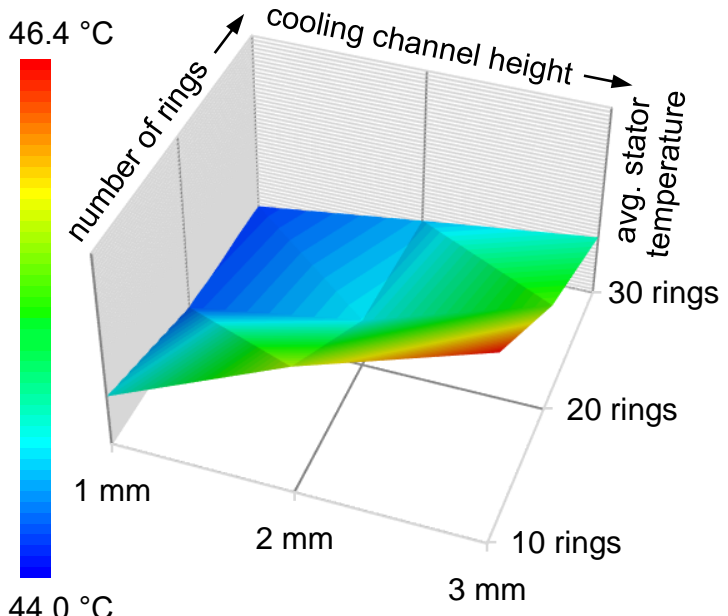

(b) meander stator cooling sleeve

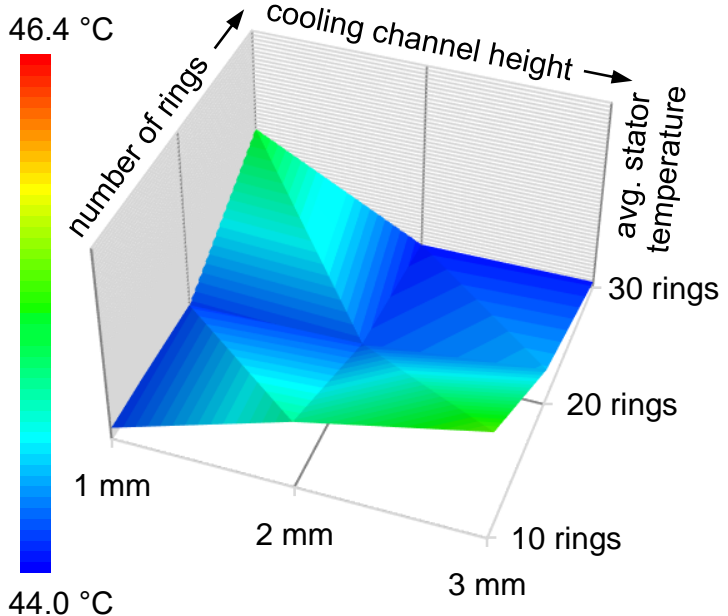

(c) S-meander stator cooling sleeve

Fig. 6: Average stator temperature through a parameter variation of a helix shaped (Fig. 6 a)), meander shaped (Fig. 6 b)) and S-meander shaped (Fig. 6 c)) stator cooling sleeve's number of rings and channel height. 
might be a second sweet spot at about 15 rings and less than $1 \mathrm{~mm}$ channel height. Interestingly, the graph looks comparable to the helix' (Fig. 6 a)). Both show a valley from the very left to the very right and a surprisingly bad result for the thinnest cooling channel at $1 \mathrm{~mm}$ and 30 rings (45.15 ${ }^{\circ} \mathrm{C}$ for the S-meander).

\section{SUMMARY}

The continuous increase in productivity of machine tools requires more and more effective cooling systems. This is especially true for motorized spindles, as these core components combine high power density with extreme precision. The spindle's fluid cooling already absorbs the largest part of the generated heat, although its flow geometry can still be vastly improved.

Therefore, an evaluation system for fluid cooling systems in motorized spindles was developed in chapter 2 . The evaluation is based on three pillars: efficiency, influence on the machine's precision and effectiveness. The efficiency was quantified through the pressure drop between the inlet and the outlet of the fluid circulation system. Its influence to the machine's precision was evaluated based on the thermal asymmetry it introduces into the spindle's housing. Furthermore, its effectiveness was quantified based on the average temperature of the electric motor's stator inside the spindle. The observation was based on a validated coupled thermal/fluid-mechanical simulation model which was published earlier.

Chapter 3 presented an analysis of different stator cooling sleeve designs. The best designs - the helix and the meander shaped cooling sleeve - were then compared in detail. Based on the advantages of these concepts, a socalled S-meander shaped sleeve was developed. It combines the high fluid velocity of the helix and the lower pressure drop of the meander shaped cooling sleeve.

These three sleeves were then compared extensively through a geometry variation study in chapter 4 . In total 27 simulation models with different geometric parameters (channel width and/or number of rings) were conceptualized for the three basic sleeve forms from chapter 3 and compared based on the criteria derived in chapter 2 . The meander shaped stator cooling sleeve generally showed the lowest pressure drop. However, the S-meander shaped stator cooling sleeve yielded the lowest thermal asymmetry and stator temperatures.

In conclusion, the introduced three pillar evaluation concept is a suitable tool for reviewing fluid cooling systems in motorized spindles. However, future iterations of the model should feature an observation of the stator's winding overhang temperature. With precise winding overhang temperatures, this evaluation concept and the researched fluid circulation systems can be transferred to fluid cooled electric motors in general. Additionally, the 3D graphs in chapter 4 suggest that the sleeves can be developed further to reach even lower pressure drops, thermal asymmetries and stator temperatures.

\section{REFERENCES}

[Abele 2010] Abele, E. et al. Machine tool spindle units, CIRP Annals, 2010, Volume 59, Issue 2, pp 781-802. ISSN 0007-8506

[Anderl 2014] Anderl, R. and Binde, P. Simulationen mit NX - Kinematik, FEM, CFD, EM und Datenmanagement mit zahlreichen Beispielen fuer NX 9, 2014, Muenchen: Carl Hanser Verlag. ISBN 3446439218
[Brecher 2018] Brecher, C. et al. Metrological analysis of a mechatronic system based on novel deformation sensors for thermal issues in machine tools, Procedia CIRP, 2018, Volume 77, pp 517-520, ISSN 2212-8271

[Cuiping 2017] Cuiping, L. et al. Optimal design of cooling system for water cooling motor used for mini electric vehicle, proceedings of the 20th International Conference on Electrical Machines and Systems (ICEMS): Sydney, August 11-14, 2017

[Ganesh 2012] Ganesh, K. M. M. et al. CFD Analysis of cooling channels in built-in motorized high speed spindle, IRACST- Engineering Science and Technology: An International Journal (ESTIJ), 2012 Volume 2, pp 238-244, ISSN 2250-3498

[Horejs 2012] Horejs, O. et al. Advanced Modelling of Thermally Induced Displacements and Its Implementation

into Standard CNC Controller of Horizontal Milling Center, Procedia CIRP, 2012, Volume 4, pp 67-72. ISSN 22128271

[Koch 2017] Koch, L. et al. Coupled thermal and fluid mechanical modeling of a high speed motor spindle, Energy Efficiency in Strategy of Sustainable Production III, 2017, Volume 871, Applied Mechanics and Materials, Trans Tech Publications Ltd, pp 161-168

[Koch 2021] Koch, L. et al. Thermal Asymmetry Analysis of Motorized Spindles, In: M. Sulitka and S. Ihlenfeldt, ed. proceedings of the Conference on Thermal Issues in Machine Tools: Prague, April 19-21, 2021

[Liu 2016] Liu, K. et al. Modeling and compensation for spindle's radial thermal drift error on a vertical machining center, International Journal of Machine Tools and Manufacture, 2016, Volume 105, pp 58-67. ISSN 08906955

[Pechanek 2012] Pechanek, R. and Buzek, L. Analyzing of Two Types Water Cooling Electric Motors Using Computational Fluid Dynamics, proceedings of the 15th International Power Electronics and Motion Control Conference (EPE/PEMC): Novi Sad, September 4-6, 2012

[Satrustegui 2017] Satrustegui, M. et al. Design criteria for water cooled systems of induction machines, Applied Thermal Engineering, 2017, Volume 114, pp 1018-1028, ISSN 1359-4311

[Weber 2016] Weber, J. et al. Thermal impact of different cooling sleeve's flow geometries in motorized high speed spindles of machine tools, proceedings of the 9th FPNI Ph.D. Symposium on Fluid Power (FPNI2016): Florianopolis, October 26-28, 2016

[Yang 2016] Yang, C. et al. Design and Analysis of Cycling Oil Cooling in Driving Motors for Electric Vehicle Application, proceedings of the IEEE Vehicle Power and Propulsion Conference (VPPC): Hangzhou, October 17-20, 2016

[Zhen-nan 2011] Zhen-nan, Y. et al. Simulative analysis of traction motor cooling system based on CFD, proceedings of the 11. International Conference on Electric Information and Control Engineering: Wuhan, April 15-17, 2011, pp. 746-749 